\title{
Asymptomatic infection outcomes for brucellosis: A meta-analysis
}

\author{
Huixin Yang ${ }^{1}$, Xiangyi Zhang ${ }^{1}$, Jing $\mathrm{Hu}^{1}$, Siwen Zhang ${ }^{1}$, Taijun Wang ${ }^{1}$, Mujinyan Li $^{1}$, \\ Fande $\mathrm{Li}^{1}$, Shuqi $\mathrm{An}^{1}$, Cui Zhang ${ }^{1}$, and Qing Zhen ${ }^{1}$ \\ ${ }^{1}$ Affiliation not available
}

October 22, 2020

\begin{abstract}
Abstract Background A Brucella leak occurred at the Lanzhou Biopharmaceutical Factory in China. As of September 14, 2020, 96 brucella asymptomatic infections have been detected, while little is known about them due to lack of the follow-up study. Methods To synthesise the evidence of the effectiveness of asymptomatic brucellosis infection. Multiple databases Were searched and inclusion Criteria included participants were newly infected with Brucella, and had a positive serum agglutination test (SAT[?]100),no symptoms, such as fever, sweating, malaise; Papers were quality assessed used the Cochrane risk bias tool. Results Meta-analyses were performed for risk difference and standardised mean difference of all asymptomatic brucellosis infection. we conducted a meta-analysis of the published literatures. A total of 56 asymptomatic infections were included in, male adults accounted for the majority of the cases, and both occupational and non-occupational groups had asymptomatic infection; $20 \%$ (95\% CI:0\%-55\%) of subjects had positive outcomes and the follow up time ranged from 1-12 months in metaanalysis. Conclusions Meta-analysis suggests that both occupational and non-occupational groups have asymptomatic infection and male adults account for the majority and most of the subjects showing positive outcome exposed to sheep or unpasteurized dairy products. Key words: Brucellosis, asymptomatic infection, meta-analysis
\end{abstract}

\section{Hosted file}

manuscript.pdf available at https://authorea.com/users/369510/articles/488327-asymptomaticinfection-outcomes-for-brucellosis-a-meta-analysis 


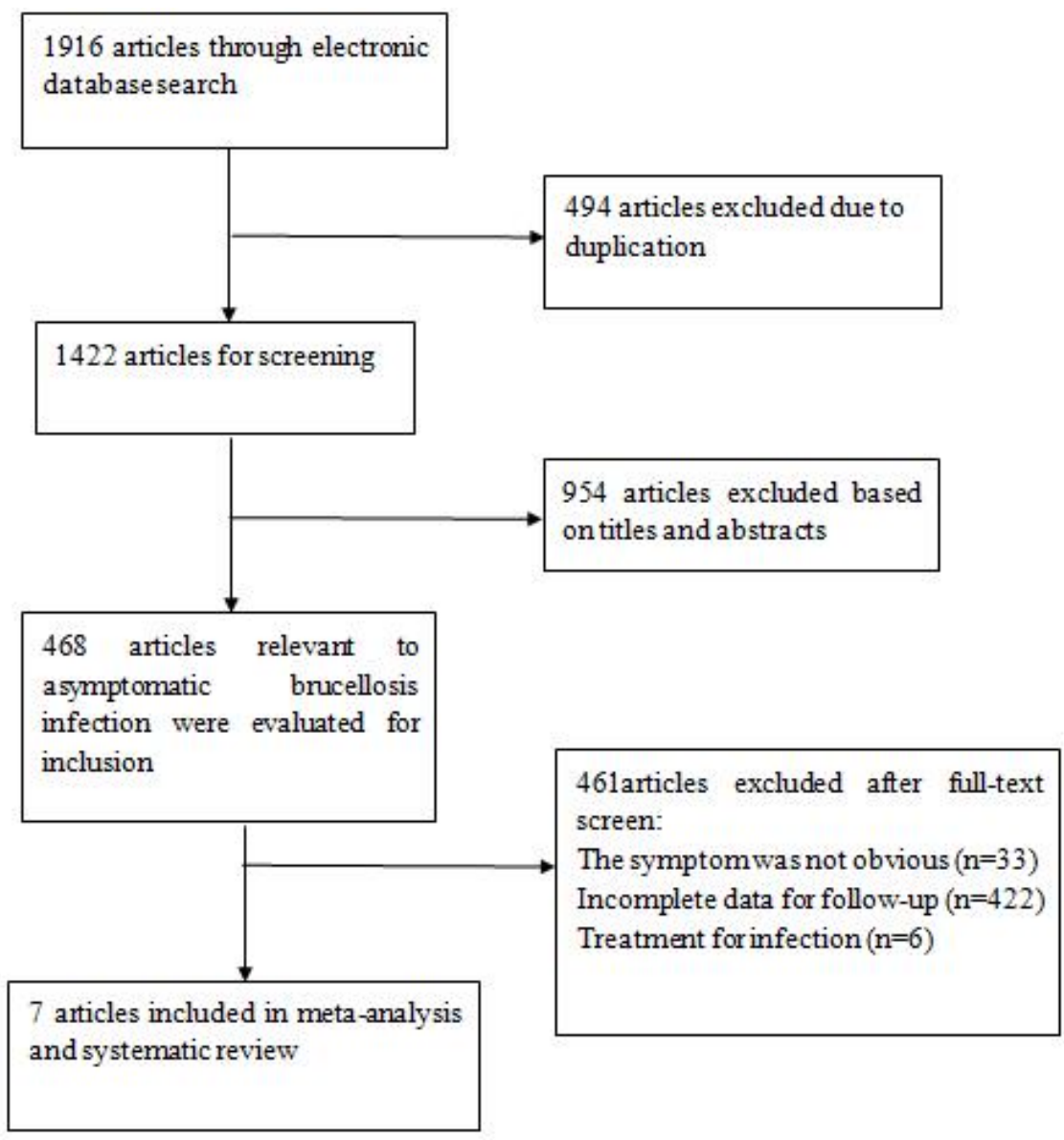

FIG.1.Flow chart of searching

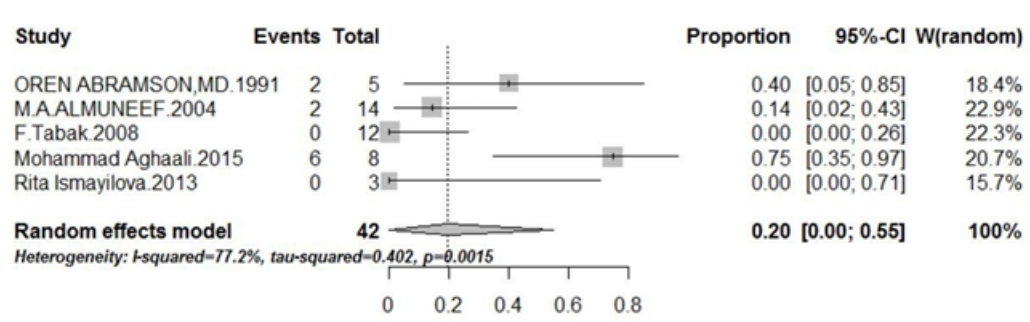

FIG.2.Proportion of positive outcome among asymptomatic infection, weighted by random-effects analysis. $\mathrm{CI}=$ confidence interval. 


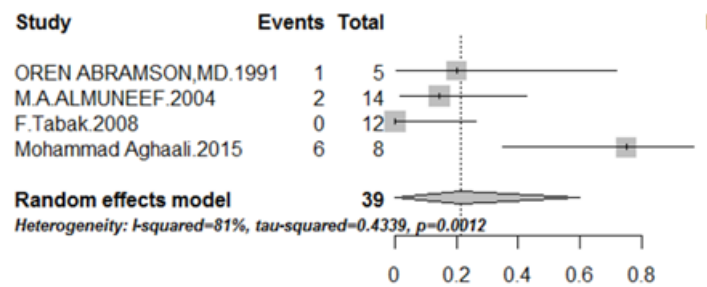

Proportion $\quad 95 \%-\mathrm{Cl}$ W(random)

$0.20[0.01 ; 0.72] \quad 22.0 \%$ $0.14[0.02 ; 0.43] \quad 27.0 \%$ $0.00[0.00 ; 0.26] \quad 26.4 \%$ $0.75[0.35 ; 0.97] \quad 24.6 \%$

$0.21[0.00 ; 0.60] \quad 100 \%$

FIG.3.Proportion of symptom developed among asymptomatic infection, weighted by random-effects analysis. $\mathrm{CI}=$ confidence interval.

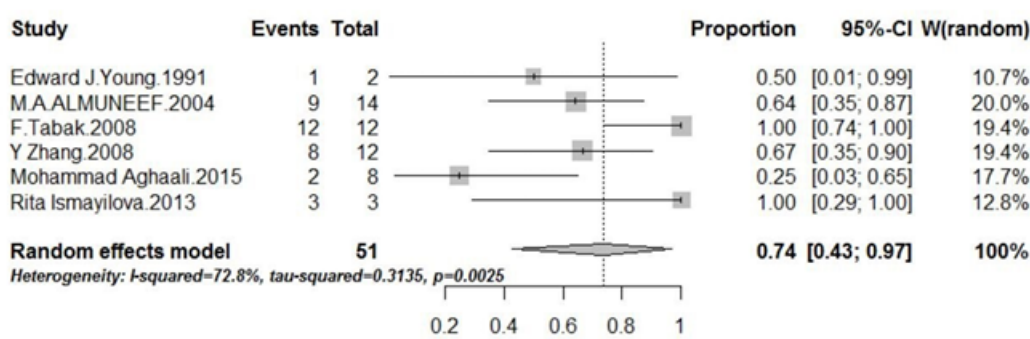

FIG.4.Proportion of negative outcome among asymptomatic infection, weighted by random-effects analysis. $\mathrm{CI}=$ confidence interval.

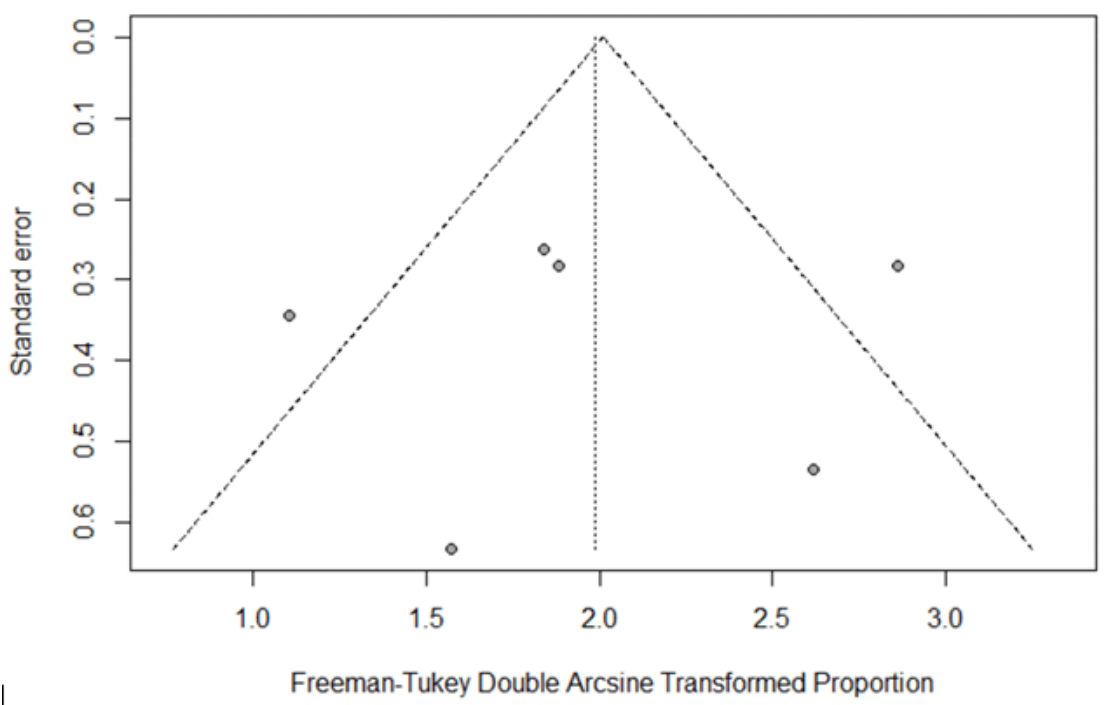

FIG.5. A funnel plot of asymptomatic infection in patients with negative outcomes 